(C) 2020 IEEE

\title{
Direct Arm Energy Control in Modular Multilevel Converter Under Unbalanced Grid Conditions
}

M. Utvic and D. Dujic

This material is posted here with permission of the IEEE. Such permission of the IEEE does not in any way imply IEEE endorsement of any of EPFL's products or services. Internal or personal use of this material is permitted. However, permission to reprint / republish this material for advertising or promotional purposes or for creating new collective works for resale or redistribution must be obtained from the IEEE by writing to pubs-permissions@ieee. org. By choosing to view this document, you agree to all provisions of the copyright laws protecting it. 


\section{Direct Arm Energy Control in Modular Multilevel Converter Under Unbalanced Grid Conditions}

\author{
Milan Utvic \\ Power Electronics Laboratory \\ École polytechnique fédérale de Lausanne \\ Lausanne, Switzerland \\ milan.utvic@epfl.ch
}

\author{
Drazen Dujic \\ Power Electronics Laboratory \\ École polytechnique fédérale de Lausanne \\ Lausanne, Switzerland \\ drazen.dujic@epfl.ch
}

\begin{abstract}
This paper studies the behaviour of the gridconnected modular multilevel converter under unbalanced grid conditions in terms of the energy imbalances within the converter, and proposes a novel approach for dealing with imbalances. Since the energy content within the arms of modular multilevel converter depends on the interaction between the terminal and internal voltages and currents, standard energy control methods, designed mainly for balanced grid conditions require improvement. This paper proposes a novel approach for the direct arm energy control, valid for both balanced and unbalanced grid conditions. The approach is characterized with its simple implementation, minimization of the current stress imposed on the converter, as well as with minimization of the risk of overmodulation. Theoretical foundation for mitigating the problem are supported with the simulation results, thus verifying the validity and performance of the proposed control concepts.

Index Terms - modular multilevel converter, energy balancing, unbalanced grid conditions, direct arm energy control
\end{abstract}

\section{INTRODUCTION}

Ever since its introduction in [1] modular multilevel converter (MMC) has become standard solution in many demanding power applications. The most prominent application of MMC is in high voltage DC (HVDC) transmission systems, where contemporary converter topologies are mostly based on MMC, owing to the advantages of the voltage source converters (VSCs) over the standard line-commutated converters (LCCs), such as independent reactive and active power control, fast transient response, ability to supply weak networks, and black start capability [2]. Apart from the advantages offered by the nature of VSCs, MMC has some additional prominent features, such as the ease of scalability, modularity, redundancy, high efficiency, elimination of the bulky transformers, filters and DC link capacitor [3], making the MMC preferable VSC solution for the high voltage, high power transfer. Except for the ultra-high DC currents, when thyristor-based LCCs are used, owing to the aforementioned advantages, MMC is becoming preferable solution for the new HVDC projects. Both academia and industry are showing interest in the application of MMC in various other areas, such as flexible AC transmission systems (FACTS) [4]-[6], breaker-less medium voltage DC (MVDC)-shipboard systems [7], offshore wind farms MVDC collection grids [8], integration of the energy storage systems, etc.
Contrary to the another prominent multilevel topologycascaded H-bridge (CHB) converters, which have their submodules (SMs) externally powered, SMs inside an MMC are dynamically charged and discharged only by the converter currents. Consequently, besides standard control loops being present in CHB converters, MMC needs additional layers of control, which maintain the voltage (energy) levels inside the SMs around the predefined values. In that sense, it is necessary to perform the inter-arm energy balancing, which ensures equal energy content of each one of the MMC arms, as well as the intra-arm energy balancing, where the focus is on the equal distribution of energy among the SMs within an arm.

While intra-arm voltage (energy) balancing methods are various and well studied in the literature [1], [9], [10], they are not influenced by the grid conditions, nor by the methods used for the inter-arm energy balancing. On the other hand, inter-arm energy balancing relies on the interaction between the internal and terminal MMC voltages and currents, and is therefore dependent on the grid conditions.

Owing to its modularity, redundancy and good dynamic performance, MMC is perceived as a robust converter, able to remain in operation under various abnormal conditions, such as tolerances in the components, failure of a SM, unbalanced grid conditions, etc. Failure of one or several SMs within an arm should not prevent normal operation of an MMC, however the energy content of the corresponding arm changes accordingly. Contrary to the arm energy balancing methods, which ensure equal distribution of the total energy among the MMC arms, this paper introduces the direct arm energy control (DAEC) method, which enables independent control of the arm energies.

Faults in a three-phase (3PH) electric grid give rise to the unbalanced grid conditions, when three-phase grid voltages do no longer constitute symmetrical three-phase system, and are usually analysed by means of its direct, inverse and zero sets of symmetrical components. On a per-phase level, grid voltages during the imbalances generally have different magnitudes, and their mutual phase disposition deviate from the one in a symmetrical $3 \mathrm{PH}$ system $(2 \pi / 3)$. This paper explores a negative influence that the grid unbalances have on the MMC arm energies, and proposes a simple and efficient DAEC method valid for both balanced and unbalanced grid 
conditions. The results presented in this paper do not depend on the intra-arm voltage (energy) balancing method being employed.

Control method ensuring energy balancing among the arms of an MMC was introduced in [11], and has also been well studied in the literature [12]-[14]. However, these concepts are applicable to the symmetrical grid conditions. Regarding the arm energy control under asymmetrical grid conditions, several references are also available, proposing different control methods aiming to ensure inter-arm energy balancing [15]-[17]. While the energy balancing methods proposed in these papers mostly employ decomposition of the control variables into the positive/negative/zero sequence components and manipulation with the matrices of state-variables in order to obtain energy balancing references, method proposed within this paper is developed on a per-phase basis, generating energy control references in a simple manner, and ensuring a minimal current stress to the converter as well as a minimal risk of overmodulation.

\section{DIRECT ARM ENERGY CONTROL}

\section{A. Motivation}

The goal of the internal balancing control is to ensure that the energy stored within the SMs of each arm corresponds to the reference value. In normal operation, the number of SMs per arm is equal in all converter arms, and sum of their voltages is kept around the rated DC voltage, $V_{\mathrm{DC}}^{\text {nom }}$. However, in case of failure of a SM within an arm, remaining SMs are surcharged so that their sum voltage remains $V_{\mathrm{DC}}$, as in normal operation. In this case, the arm with failed SM contains more energy stored compared to the other arms. Therefore, each arm should be equipped with a dedicated energy controller in order to ensure direct control of its energy content.

Energy imbalances within an MMC also occur as a result of the abnormal grid conditions, such as faults and voltage imbalances, as it will be demonstrated later in the paper. Imbalance between the arms energies might also occur naturally in normal operation, due to parasitic circulating current at fundamental frequency, that appears in MMC as a result of inductance tolerances [18]. Finally, using a dedicated energy controllers is also important in order to gain asymptotic stability of the closed-loop controlled MMC, as suggested in [19].

Owing to the energy control mechanisms, discussed further in this section, direct arm energy control is performed

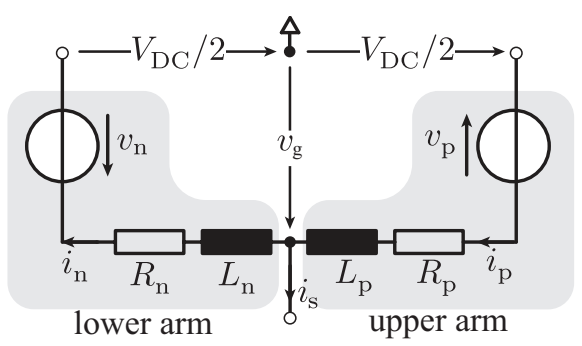

Fig. 1. Per-phase equivalent circuits of an MMC phase-leg through the so-called arm-differential $(\Delta)$ energy control and arm-sum $(\Sigma)$ energy control. The former control mechanism ensures that the energy difference between the upper (p) and lower (n) arm (c.f. Fig. 1) of a single phaseleg $W_{\Delta}=W_{\mathrm{p}}-W_{\mathrm{n}}$ matches the difference between the arm energy references $W_{\Delta}^{*}=W_{\mathrm{p}}^{*}-W_{\mathrm{n}}^{*}$. The latter control mechanism ensures that the sum energy of the belonging arms $W_{\Sigma}=W_{\mathrm{p}}+W_{\mathrm{n}}$ matches the sum of the arm energy references $W_{\Sigma}^{*}=W_{\mathrm{p}}^{*}+W_{\mathrm{n}}^{*}$. Consequently, with two energy controllers per phase-leg ( $\Delta$ and $\Sigma$ energy control), energy content within each arm is controlled directly.

\section{B. MMC voltages and currents as the arm energy control variables}

To identify the control variables for the direct arm energy control, one should refer to the equivalent electrical circuit of a phase-leg of an MMC (c.f. Fig. 1), and derive arm power equations accordingly. Introducing the arm-common and arm-differential voltages and currents, $\left(v_{\mathrm{c}}, i_{\mathrm{c}}\right)$ and $\left(v_{\mathrm{s}}, i_{\mathrm{s}}\right)$, respectively, defined by the expressions in (1), the appropriate instantaneous power equations for the upper and the lower arms can be derived (2)-(3).

$$
\begin{aligned}
& i_{\mathrm{c}}=\left(i_{\mathrm{p}}+i_{\mathrm{n}}\right) / 2 \quad i_{\mathrm{s}}=i_{\mathrm{p}}-i_{\mathrm{n}} \\
& v_{\mathrm{c}}=\left(v_{\mathrm{p}}+v_{\mathrm{n}}\right) / 2 \quad v_{\mathrm{s}}=\left(v_{\mathrm{n}}-v_{\mathrm{p}}\right) / 2
\end{aligned}
$$

Based on (2)-(3) the expressions for the arm-differential $p_{\Delta}$ and arm-sum power $p_{\Sigma}$ can be derived. These two power terms are time derivatives of the arm-differential $W_{\Delta}$ and arm-sum $W_{\Sigma}$ energies, and are consequently used in $\Delta$ and $\Sigma$ energy control.

$$
\begin{gathered}
p_{\mathrm{p}}=v_{\mathrm{p}} i_{\mathrm{p}}=\left(v_{\mathrm{c}}-v_{\mathrm{s}}\right)\left(i_{\mathrm{c}}+i_{\mathrm{s}} / 2\right) \\
p_{\mathrm{n}}=v_{\mathrm{n}} i_{\mathrm{n}}=\left(v_{\mathrm{c}}+v_{\mathrm{s}}\right)\left(i_{\mathrm{c}}-i_{\mathrm{s}} / 2\right) \\
p_{\Delta}=p_{\mathrm{p}}-p_{\mathrm{n}}=v_{\mathrm{c}} i_{\mathrm{s}}-2 v_{\mathrm{s}} i_{\mathrm{c}} \\
p_{\Sigma}=p_{\mathrm{p}}+p_{\mathrm{n}}=2 v_{\mathrm{c}} i_{\mathrm{c}}-v_{\mathrm{s}} i_{\mathrm{s}}
\end{gathered}
$$

Applying the Kirchhoff's voltage law to the upper and lower arm (c.f. Fig. 1), one can obtain equations relevant for the arm-differential current $i_{\mathrm{s}}$ and arm-common current $i_{\mathrm{c}}$ control, where $L_{\Sigma}=L_{p}+L_{n}$, and assuming $L_{\Delta}=L_{p}-L_{n}=0$.

$$
v_{\mathrm{s}}-v_{\mathrm{g}}=\frac{L_{\Sigma}}{4} \frac{d i_{\mathrm{s}}}{d t} \quad \text { (6) } \quad V_{\mathrm{DC}}-2 v_{\mathrm{c}}=L_{\Sigma} \frac{d i_{\mathrm{c}}}{d t}
$$

From (6)-(7) one can conclude that the arm-common current control and arm-differential current control on a per-phase level are decoupled from each other, provided that the upper and lower arm inductances match, i.e. $L_{\Delta}=0$. It can also be noticed that the arm-differential voltage $v_{\mathrm{s}}$ has the AC nature, and is in charge of $i_{\mathrm{s}}$ current control, which corresponds to the $\mathrm{AC}$ terminal current (grid current). The arm-common voltage $v_{\mathrm{c}}$ has the DC nature, and is in charge of the arm-common current $i_{\mathrm{c}}$ control. This current component consists of the DC part, which ideally corresponds to $i_{\mathrm{DC}} / 3$ in a $3 \mathrm{PH} \mathrm{MMC}$, and additional circulating current components, which circulate among the MMC phase-legs, and are not observed at the MMC terminals. 


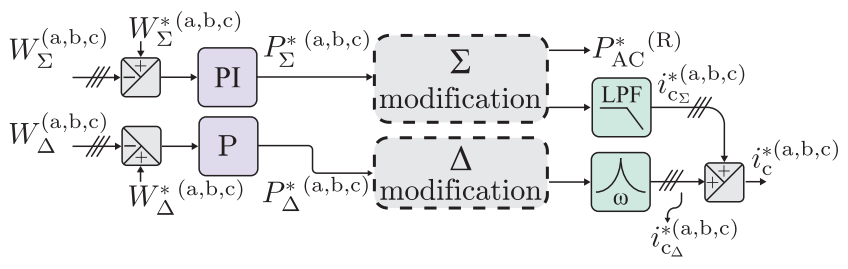

Fig. 2. Control structure of $W_{\Sigma}$ and $W_{\Delta}$ arm energies

Analyzing the power terms in (4), one can conclude that the average value of the arm-differential power $p_{\Delta}$ over a fundamental period has a nonzero value in case when a fundamental frequency component exists in the arm-common current $i_{\mathrm{c}}$. Therefore, arm-differential energy $W_{\Delta}$ can be controlled by introducing a fundamental frequency component $i_{\mathrm{c} \Delta}$ into the arm-common current $i_{\mathrm{c}}$. On the other hand, (5) shows that the arm-sum energy $W_{\Sigma}$ depends on the difference between the average DC power (product $v_{\mathrm{c}} i_{\mathrm{c}}$ ) and average $\mathrm{AC}$ power (product $v_{\mathrm{s}} i_{\mathrm{s}}$ ). Thus arm-sum energy is controlled by controlling the power differences on a per-phase basis.

\section{Novel ENERgy CONTROL SCHEME FOR UNBALANCED GRID CONDITIONS}

Principle of $W_{\Sigma}$ and $W_{\Delta}$ control has been explained in the previous section. In terms of control, it is based on a feedback P/PI controllers (c.f. Fig. 2), yielding necessary power references $P_{\Sigma}^{*}$ and $P_{\Delta}^{*}$, which are further transformed to the appropriate arm-common current references $i_{\mathrm{c} \Sigma}^{*}$ and $i_{\mathrm{c} \Delta}^{*}$. Current components generated for the purpose of energy control have to remain unobserved at both converter terminals. This restriction, as well as the fact that the grid voltages are unbalanced are the key challenges in MMC energy control that this paper addresses.

Energy balancing mechanisms in [15]-[17] rely on the decomposition of unbalanced grid voltages into the positive/negative/zero sequence $3 \mathrm{PH}$ systems, and generation of a corresponding current components for the balancing purpose. The approach used in this paper is derived on a per phaselevel, omitting the need for the P/N/0 decomposition, which results in simpler implementation, minimization of the current stress imposed on the converter, as well as minimization of the risk of overmodulation. As previously mentioned DAEC relies on the two control mechanisms: $\Delta$ and $\Sigma$ energy control.

\section{A. Arm-differential $(\Delta)$ energy control}

Arm-differential power $p_{\Delta}$ depends on the interaction of the arm-differential voltage $v_{\mathrm{s}}$ and introduced fundamental frequency component into the arm-common current $i_{\mathrm{c} \Delta}$. From (4) the average arm-differential power over a fundamental period equals:

$$
P_{\Delta}=V_{\mathrm{s}} I_{\mathrm{c} \Delta} \cos (\phi)
$$

where the $V_{\mathrm{s}}$ denotes magnitude of the phase voltage, $I_{\mathrm{c} \Delta}$ denotes magnitude of the introduced fundamental frequency armcommon current component, whereas $\phi$ denotes respective phase disposition among the two variables. Arm-differential voltage $v_{\mathrm{s}}$ is determined by the grid conditions, and is in charge of the grid (arm-differential) current $i_{\mathrm{s}}$ control. In order to realize the arm-differential power reference $P_{\Delta}^{*}$ with the lowest possible magnitude of the fundamental frequency armcommon current $I_{\mathrm{c} \Delta}$, voltage and current should be in phase. However, there are two major restrictions that make this task non-trivial:

- generated fundamental frequency arm-common currents $i_{\mathrm{c} \Delta}$ should sum-up to zero among the phase-legs in order not to be observed at the DC terminals of an MMC;

- arm-differential voltages are not balanced among the phases, and it is necessary to determine the magnitude and phase of each one of them.

To meet the first requirement, arm-common current references should be modified, so as to sum-up to zero. This modification, as well as criterion being used for obtaining new set of references, is illustrated in the Fig. 3. Namely, for each phase, $\mathrm{P}$ controller yields a power reference $P_{\Delta}^{*}$, which further divided by the arm-differential voltage magnitude $V_{\mathrm{s}}$ of the respective phase, yields the magnitude of the fundamental frequency arm-common current reference $I_{\mathrm{c} \Delta}^{* *}$ (green vectors in Fig. 3), assuming zero phase-shift with respect to the armdifferential voltage in that phase. Thus obtained current vectors generally have arbitrary magnitudes, and they do not sum-up to zero, so there is a need for their modification. Criterion being used for reference modification is that the modified set of references should sum-up to zero, as well as that the deviation function $A^{2}$ (c.f. Fig. 3.b) is at its minimum. In order words, new set of references should have lowest possible deviation from the original one, providing zero-sum at the same time. Thorough mathematical derivations are omitted here, and only the final conclusion in being presented. Namely, a new set of current references (purple vectors in Fig. 3) is obtained by finding the average (zero) component of the original set of current vectors $\vec{I}_{\mathrm{c} \Delta}^{* *}$, followed by its subtraction from the respective current vectors. And indeed, if any $3 \mathrm{PH}$ signal is represented by $\alpha \beta 0$ components, the closest possible approximation of that signal under a zero-sum constraint is obtained when only zero component is removed.

Another challenge to be overcame is related to the identification of the phase-voltage magnitude and phase, necessary in order to generate proper current references. For that purpose,

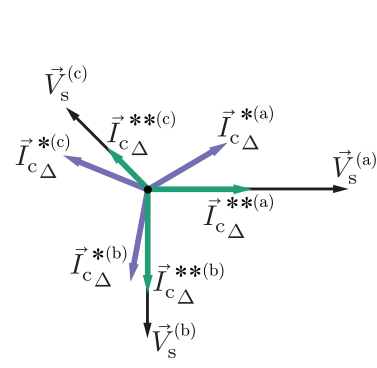

a)

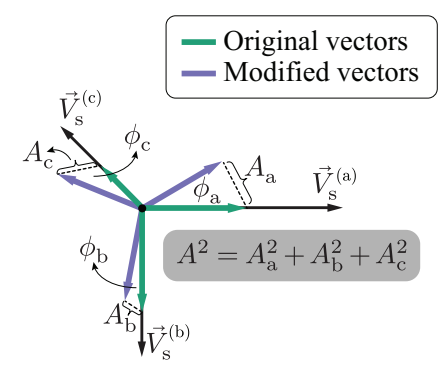

b)
Fig. 3. Arm-differential voltage vectors with the arm-common current vectors a) original and modified set of current references; b) deviation function $A^{2}$ as a criterion for the reference modification 

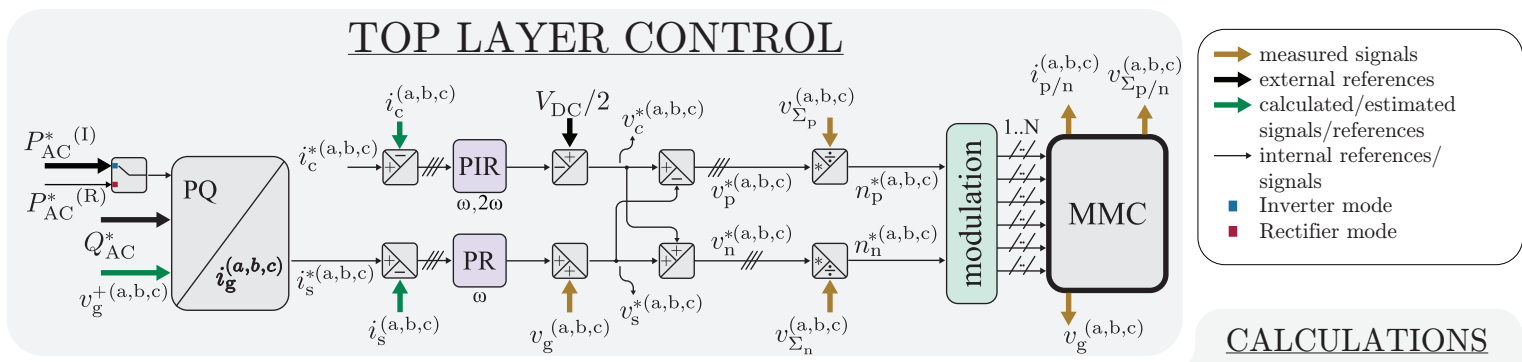

ENERGY CONTROL
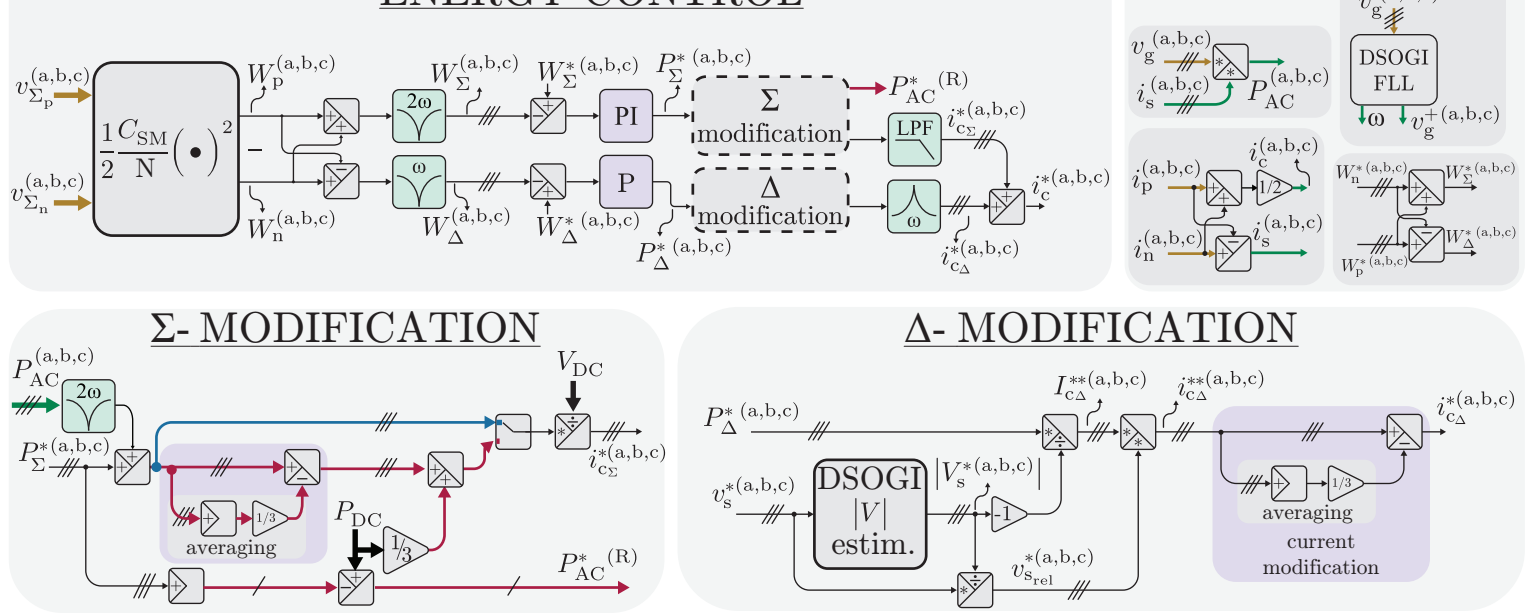

Fig. 4. Overall control structure of an MMC. $\Sigma$ and $\Delta$ energy control schemes (middle); $\Sigma$ and $\Delta$ modification blocks (bottom).

DSOGI based quadrature-signal generator [20] is used to generate signal in quadrature $v_{\mathrm{s}, \mathrm{q}}$, further used for calculation of the voltage magnitude $V_{\mathrm{s}}$ in each phase, and to obtain a relative value of the voltage signal $v_{\text {srel }}$, according to the following equations:

$$
V=\sqrt{\left(v_{\mathrm{s}}^{*}\right)^{2}+\left(v_{\mathrm{s}, \mathrm{q}}^{*}\right)^{2}} \quad \text { (9) } \quad v_{\mathrm{s}, \mathrm{rel}}^{*}=v_{\mathrm{s}}^{*} / V
$$

The whole block that generates adequate arm-common current references for the purpose of $\Delta$ energy control is illustrated in Fig. 4, and labeled as $\Delta$-modification.

\section{B. Arm-sum $(\Sigma)$ energy control}

Arm-sum energy variation of an MMC phase depends on the difference between the products $2 v_{\mathrm{c}} i_{\mathrm{c}}$ and $v_{\mathrm{s}} i_{\mathrm{s}}$, according to

TABLE I

TEST CONVERTER PARAMETERS

\begin{tabular}{lcc}
\hline \hline \multicolumn{1}{c}{ Parameter } & Label & Value \\
\hline Line voltage & $U_{\mathrm{n}}$ & $3.3 \mathrm{kV}$ \\
Apparent power & $S_{\mathrm{n}}$ & $250 \mathrm{kVA}$ \\
Grid frequency & $f_{\mathrm{n}}$ & $50 \mathrm{~Hz}$ \\
Number of SMs per arm & $N_{\mathrm{SM}}$ & 8 \\
SM capacitance (tolerance $\pm 5 \%)$ & $C_{\mathrm{SM}}$ & $2.25 \mathrm{mF}$ \\
Arm inductance & $L_{\mathrm{br}}$ & $2.5 \mathrm{mH}$ \\
Arm resistance & $R_{\mathrm{br}}$ & $20 \mathrm{~m} \Omega$ \\
Switching frequency & $f_{\mathrm{sw}}$ & $1000 \mathrm{~Hz}$ \\
\hline \hline
\end{tabular}

(5). Voltage $v_{\mathrm{c}}$ closely corresponds to the $V_{\mathrm{DC}} / 2$, whereas the arm-common current in the steady state corresponds to $i_{\mathrm{DC}} / 3$. Therefore, the product $2 v_{\mathrm{c}} i_{\mathrm{c}}$ represents the active power that a phase-leg exchanges with the DC terminals. On the other hand, the product $v_{\mathrm{s}} i_{\mathrm{s}}$ represents an active power of a phase-leg exchanged with its AC terminals. Depending on the operating mode of an MMC, either an active power delivered to the DC terminals or an active power delivered to the AC terminals is commanded from the higher instance, and consequently the arm-sum energy control strategy differs. Illustration of the $\Sigma$ energy control is given in the Fig. 4 , together with the $\Sigma$ modification block, further described hereafter.

$$
p_{\Sigma}^{*}=V_{\mathrm{DC}} i_{\mathrm{c}}-v_{\mathrm{s}} i_{\mathrm{s}}
$$

According to (11), arm-sum power reference can be realized using either arm-common current $i_{\mathrm{c}}$ or arm-differential current $i_{\mathrm{s}}$. The choice of the control variable depends on the operating mode (rectifier or inverter).

1) Inverter mode: When MMC operates as an inverter, it is supposed to provide active and reactive power to the grid. In order to preserve $\Sigma$ energy at defined level, power obtained from the DC terminals should correspond to the power requirements of each individual phase-leg. In other words, each phase-leg demands the active power resulting from the $\Sigma$ energy controller output $p_{\Sigma}^{*}$, plus the active power delivered to the AC grid through that particular phase (product $v_{\mathrm{s}} i_{\mathrm{s}}$, as shown in (12).

$$
V_{\mathrm{DC}} i_{\mathrm{c}_{\Sigma}}^{*}=v_{\mathrm{s}} i_{\mathrm{s}}+p_{\Sigma}^{*}
$$




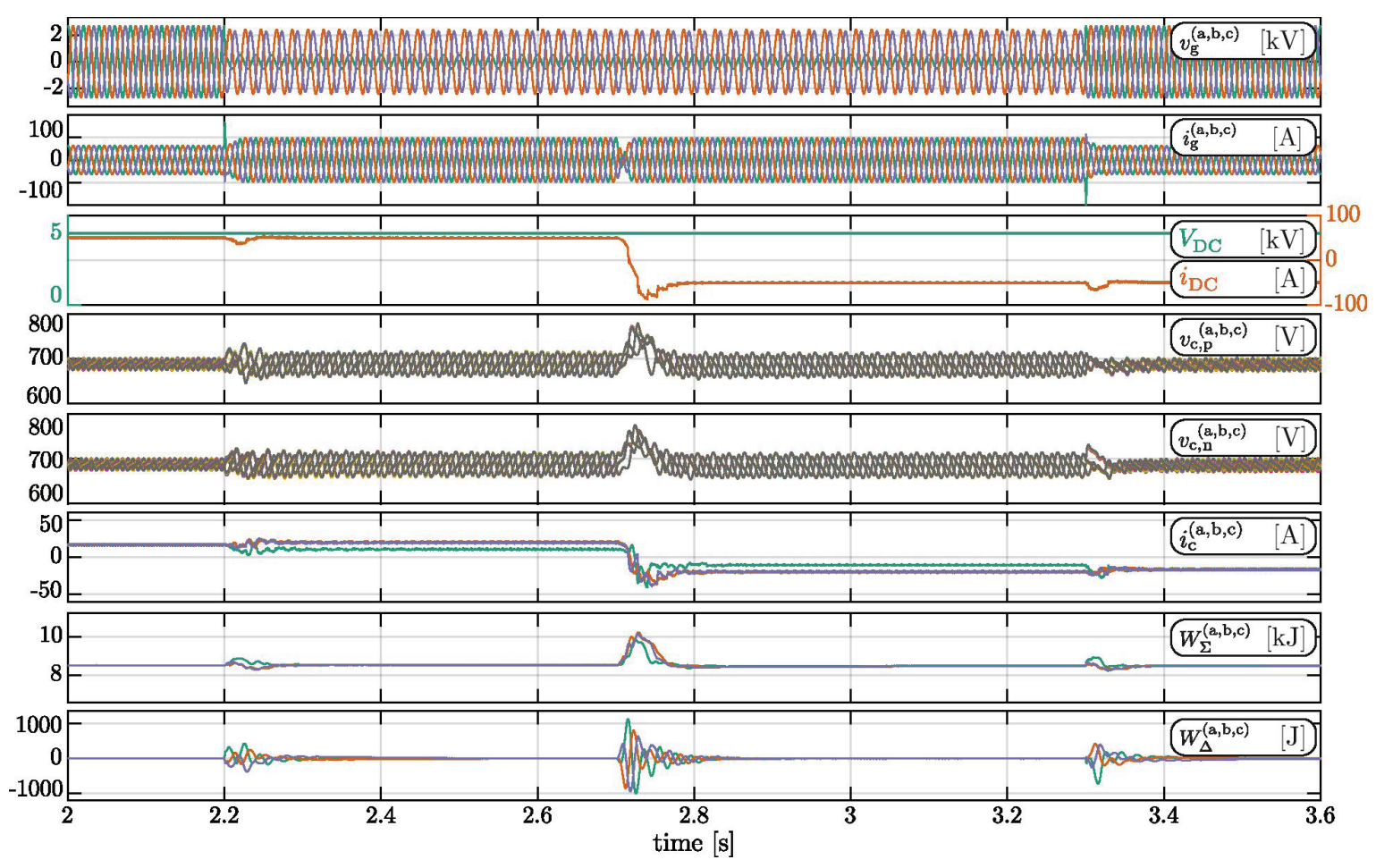

Fig. 5. Direct arm energy control performance evaluation in inverter mode of operation. Single phase-to-ground fault at $\mathrm{t}=1 \mathrm{~s}$; active power reversal under unbalanced grid conditions at $\mathrm{t}=1.6 \mathrm{~s}$; fault clearance at $\mathrm{t}=2.2 \mathrm{~s}$.

Therefore, arm-common currents are in this mode of operation controlled to correspond to the reference from (12). They have a DC nature, and their sum among the phase legs constitutes the DC terminals current. Simple control structure in this mode of operation is illustrated in Fig. 4, and relevant signals are labeled with blue color.

2) Rectifier mode: In case when an MMC operates as a rectifier, it supplies DC terminals with a certain DC voltage and current, defined by the application. In normal operation, arm-common current is $i_{\mathrm{c}}=i_{\mathrm{DC}} / 3$, and different $\Sigma$ energy controller outputs $p_{\Sigma}^{*}$ result in different requirements of the $v_{\mathrm{s}} i_{\mathrm{s}}$ product among the MMC phases, according to (11). In both symmetrical and asymmetrical grid conditions, this leads to the asymmetrical grid current $i_{\mathrm{s}}$ requirements, which is generally not permissible. Therefore, by summing the equation (13) for the three MMC phases, one obtains the $3 \mathrm{PH}$ AC power reference $P_{\mathrm{AC}}^{*}(14)$.

$$
\begin{aligned}
& p_{\Sigma}^{*}=V_{\mathrm{DC}} i_{\mathrm{c}}-v_{\mathrm{s}} i_{\mathrm{S}} \quad \Longrightarrow \quad v_{\mathrm{s}} i_{\mathrm{s}}=V_{\mathrm{DC}} i_{\mathrm{c}}-p_{\Sigma}^{*} \\
& P_{\mathrm{AC}}^{*}{ }^{(\mathrm{R})}=\sum_{x=a, b, c} v_{\mathrm{s}}^{(\mathrm{x})} i_{\mathrm{s}}^{(\mathrm{x})}=V_{\mathrm{DC}} i_{\mathrm{DC}}-\sum_{x=a, b, c} p_{\Sigma}^{*(\mathrm{x})}
\end{aligned}
$$

However, due to the fact that requested AC power $\left(P_{\mathrm{AC}}^{*}{ }^{(\mathrm{R})}\right)$ refers to the $3 \mathrm{PH}$ power, neither does it satisfy the requirements of the energy controllers $p_{\Sigma}^{*}$ in each particular phase, nor is the active AC power equal among the phases during unbalanced grid conditions. To cover the difference, an additional arm-common DC current component $\Delta i_{c_{\Sigma}}$ is introduced, with the arm-common current having the following form:

$$
i_{\mathrm{c}_{\Sigma}}=i_{\mathrm{DC}} / 3+\Delta i_{\mathrm{c}_{\Sigma}}
$$

Substituting (15) into (11) yields:

$$
\begin{gathered}
p_{\Sigma}^{*}=V_{\mathrm{DC}}\left(i_{\mathrm{DC}} / 3+\Delta i_{\mathrm{c}_{\Sigma}}\right)-v_{\mathrm{s}} i_{\mathrm{s}} \\
V_{\mathrm{DC}} \Delta i_{\mathrm{c}_{\Sigma}}=-V_{\mathrm{DC}} i_{\mathrm{DC}} / 3+v_{\mathrm{s}} i_{\mathrm{s}}+p_{\Sigma}^{*}
\end{gathered}
$$

Sum of the $i_{\mathrm{c} \Sigma}$ currents among the three phase-legs has to remain equal to the DC current $i_{\mathrm{DC}}$, leading to the condition $\sum \Delta i_{\mathrm{c}_{\Sigma}}=0$. These current components intend to cover the difference between the DC power $\left(V_{\mathrm{DC}} i_{\mathrm{DC}} / 3\right)$ and actual $\mathrm{AC}$ power $\left(v_{\mathrm{s}} i_{\mathrm{s}}\right)$ per phase, as well as to satisfy $\Sigma$ energy controller requirements $p_{\Sigma}^{*}$, as shown in (17). To generate $\Delta i_{\mathrm{c}_{\Sigma}}$ currents which sum-up to zero, the same approach is used as in the case of $i_{\mathrm{c}} \Delta$ currents, where the average (zero) value of the original references is subtracted from each one of them. This control principle is illustrated in the Fig. 4, and relevant signals for this mode of operation are labeled with red color.

Notice that in both operating modes corresponding terminal powers $\left(P_{\mathrm{DC}}\right.$ or $\left.P_{\mathrm{AC}}\right)$ are used as a feedforward signals, with the aim of improving the dynamic response of the energy controller.

\section{EVAluation of THE CONTROL METHODS}

To verify proposed control concepts, MMC with the parameters provided in Table I is simulated in PLECS. In Fig. 5 the inverter mode of operation is presented. In this mode of operation, $\mathrm{AC}$ power reference is received externally, and MMC is injecting required $\mathrm{AC}$ current into the grid. At the time instant $t=2.2 s$ single-phase-to-ground fault occurs in the grid, thus 


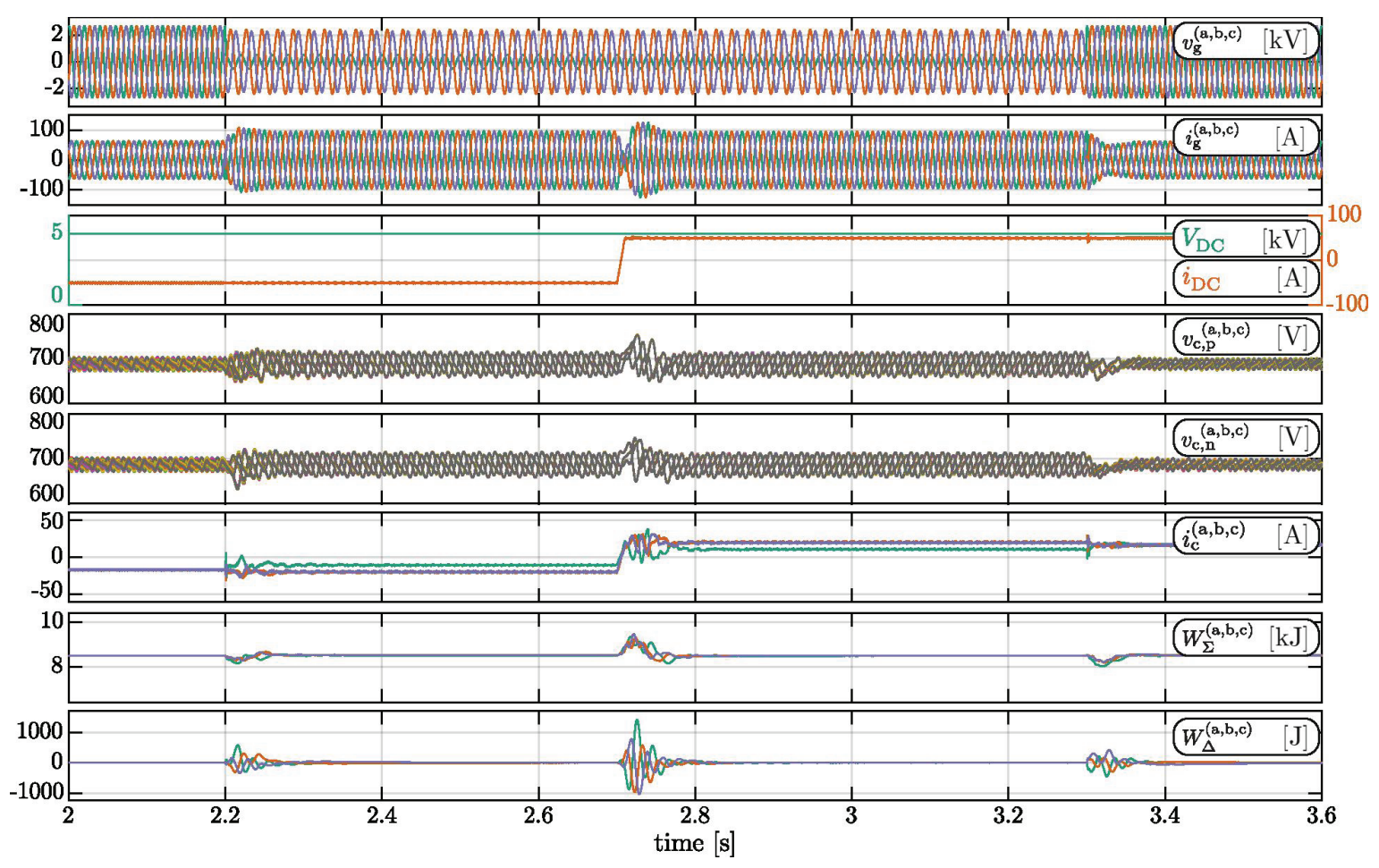

Fig. 6. Direct arm energy control performance evaluation in rectifier mode of operation; Single phase-to-ground fault at $\mathrm{t}=1 \mathrm{~s}$; active power reversal under unbalanced grid conditions at $\mathrm{t}=1.6 \mathrm{~s}$; fault clearance at $\mathrm{t}=2.2 \mathrm{~s}$.

introducing unbalanced grid conditions. The control strategy applied in this study regarding the grid current control keeps injecting symmetrical currents into the $\mathrm{AC}$ grid, with the aim of preserving the pre-fault power references, although different approaches could have been used. Nevertheless, it does not influence the performance of the energy controllers, which is the subject of the study. SMs' voltages $v_{\mathrm{c}, \mathrm{p}}^{(\mathrm{a}, \mathrm{b}, \mathrm{c})}$ and $v_{\mathrm{c}, \mathrm{n}}^{(\mathrm{a}, \mathrm{b}, \mathrm{c})}$ retain their reference value, as well as the $\Delta$ and $\Sigma$ energies. By observing the arm-common currents $i_{\mathrm{c}}^{(\mathrm{a}, \mathrm{b}, \mathrm{c})}$, one can notice an AC component, which is in charge of the $\Delta$ energy control, as well as different DC values of arm-common current among the phases, which corresponds to the theoretical considerations. Namely, due to the fact that the phases of the MMC are unequally loaded during the unbalance, arm-common current has to correspond the the power requirements of the respective phase. Additionaly, AC component of the arm-common current remains unobserved in the DC terminals current $i_{\mathrm{DC}}$. A small sag in the DC current can be noticed, which corresponds to the reaction of the $\Sigma$ energy controller to the instantaneous drop of the active power delivered to the AC grid. However, as soon as the $\mathrm{AC}$ currents are regulated to the value that generates pre-fault AC power, DC current returns to the pre-fault value.

To demonstrate validity of the proposed control concept for the bidirectional power flow, the AC active power reference $P_{\mathrm{AC}}^{*}$ is reversed at the time instant $t=2.7 \mathrm{~s}$. As in the previous case, proposed energy controllers restore the reference value of the SMs' voltages after $50 \mathrm{~ms}$. Finally, the fault is cleared at the time instant $t=3.3 \mathrm{~s}$, thus once again having demonstrated the functionality of the energy controllers under both balanced and unbalanced grid conditions.
Simulation results for the rectifier mode of operation of an MMC are presented in Fig. 6. In this mode of operation, DC power reference $P_{\mathrm{DC}}^{*}$ is obtained externally, and the control objective is to respect the power reference during all conditions. Contrary to the inverter mode of operation, DC current must not be influenced by the adverse grid conditions and internal energy control. In order to demonstrate proposed concepts, the same scenario is used as in the previous case. The performance of the energy controllers is as in the case of the inverter mode of operation, without influencing the DC current.

Since the proposed energy control algorithms are perphase based, requested arm-common currents correspond to the requirements of each phase, thus being the minimum necessary currents for the purpose of energy control. Apart from having a positive influence on the converter thermal stress, by minimizing those current components, a risk of overmodulation is also being minimized.

\section{CONClusion}

In order to have a proper operation of the converter under balanced and unbalanced grid conditions, energy stored within each arm of the converter should be kept around the reference value. This paper proposes a direct arm energy control method, composed of arm-differential energy control, and arm-sum energy control mechanisms. The aim is to have independent control over the energy stored within each one of the converter arms. Energy control mechanisms are analysed, and a novel energy control scheme, valid for both balanced and unbalanced grid conditions, is proposed. Control scheme is per-phase 
based, resulting in a minimal arm-common currents for the purpose of energy control. Finally, theoretical considerations are validated through PLECS simulations, where the performance of proposed energy control scheme is validated for both rectifier and inverter mode of operation, under balanced and unbalanced grid conditions. Although single-phase-to-ground fault is used as an example, energy control scheme is valid for any type of unbalanced conditions that might occur in a grid.

\section{ACKNOWLEDGEMENT}

This research project is part of the Swiss Competence Center for Energy Research SCCER FURIES of the Swiss Innovation Agency Innosuisse.

\section{REFERENCES}

[1] A. Lesnicar and R. Marquardt, "An innovative modular multilevel converter topology suitable for a wide power range," in 2003 IEEE Bologna Power Tech Conference Proceedings, vol. 3, Jun. 2003, 6 pp. Vol.3-.

[2] H. Saad, S. Dennetière, J. Mahseredjian, P. Delarue, X. Guillaud, J. Peralta, and S. Nguefeu, "Modular multilevel converter models for electromagnetic transients," IEEE Transactions on Power Delivery, vol. 29, no. 3, pp. 1481-1489, Jun. 2014.

[3] R. Marquardt, "Modular multilevel converters: State of the art and future progress," IEEE Power Electronics Magazine, vol. 5, no. 4, pp. 24-31, Dec. 2018.

[4] M. Pereira, D. Retzmann, J. Lottes, M. Wiesinger, and G. Wong, "Svc plus: An mmc statcom for network and grid access applications," in 2011 IEEE Trondheim PowerTech, Jun. 2011, pp. 1-5.

[5] Z. Guying, J. Daozhuo, and L. Xiaorang, "Modular multilevel converter for unified power flow controller application," in 2012 Third International Conference on Digital Manufacturing Automation, Jul. 2012, pp. 545549.

[6] J. Park, S. Yeo, and J. Choi, "Development of \pm 400 mvar world largest mmc statcom," in 2018 21st International Conference on Electrical Machines and Systems (ICEMS), Oct. 2018, pp. 2060-2063.

[7] R. Xie and H. Li, "Fault performance comparison study of a dual active bridge (dab) converter and an isolated modular multilevel dc/dc (im2dc) converter for power conversion module application in a breaker-less shipboard mvdc system," IEEE Transactions on Industry Applications, vol. 54, no. 5, pp. 5444-5455, Sep. 2018.

[8] J. Carr, D. Das, J. Li, J. Pan, S. Ebner, and O. Apeldoorn, "Modular multilevel converter for direct mvdc connection of offshore wind farms," in 2015 IEEE Energy Conversion Congress and Exposition (ECCE), Sep. 2015, pp. 976-982.

[9] M. Hagiwara, R. Maeda, and H. Akagi, "Control and analysis of the modular multilevel cascade converter based on double-star chopper-cells (mmcc-dscc)," IEEE Transactions on Power Electronics, vol. 26, no. 6, pp. 1649-1658, Jun. 2011.
[10] K. Ilves, L. Harnefors, S. Norrga, and H. Nee, "Predictive sorting algorithm for modular multilevel converters minimizing the spread in the submodule capacitor voltages," IEEE Transactions on Power Electronics, vol. 30, no. 1, pp. 440-449, Jan. 2015.

[11] A. Antonopoulos, L. Angquist, and H. Nee, "On dynamics and voltage control of the modular multilevel converter," in 2009 13th European Conference on Power Electronics and Applications, Sep. 2009, pp. 1-10.

[12] P. Münch, D. Görges, M. Izák, and S. Liu, "Integrated current control, energy control and energy balancing of Modular Multilevel Converters," in IECON 2010 36th Annual Conference on IEEE Industrial Electronics Society, Nov. 2010, pp. 150-155.

[13] J. Kolb, F. Kammerer, M. Gommeringer, and M. Braun, "Cascaded control system of the modular multilevel converter for feeding variable-speed drives," IEEE Transactions on Power Electronics, vol. 30, no. 1, pp. 349-357, Jan. 2015.

[14] M. Basić, S. Milovanović, and D. Dujić, "Comparison of two Modular Multilevel Converter Internal Energy Balancing Methods," 20th International Symposium on Power Electronics, Ee 2019, pp. 1-8, 2019.

[15] M. Schroeder, F. Mahr, J. Jaeger, and S. H. Haensel, "Energy balancing in the modular multilevel converter under unbalanced grid conditions," in 2017 19th European Conference on Power Electronics and Applications (EPE'17 ECCE Europe), Sep. 2017, P.1-P.10.

[16] J. Jung, S. Cui, Y. Lee, and S. Sul, "A cell capacitor energy balancing control of mmc-hvdc under the ac grid faults," in 2015 9th International Conference on Power Electronics and ECCE Asia (ICPE-ECCE Asia), Jun. 2015, pp. 1-8.

[17] A. E. Leon and S. J. Amodeo, "Energy balancing improvement of modular multilevel converters under unbalanced grid conditions," IEEE Transactions on Power Electronics, vol. 32, no. 8, pp. 6628-6637, Aug. 2017.

[18] R. Zeng, L. Xu, L. Yao, and S. J. Finney, "Analysis and control of modular multilevel converters under asymmetric arm impedance conditions," IEEE Transactions on Industrial Electronics, vol. 63, no. 1, pp. 71-81, Jan. 2016.

[19] L. Harnefors, A. Antonopoulos, S. Norrga, L. Angquist, and H. Nee, "Dynamic analysis of modular multilevel converters," IEEE Transactions on Industrial Electronics, vol. 60, no. 7, pp. 2526-2537, Jul. 2013.

[20] P. Rodríguez, A. Luna, R. S. Muñoz-Aguilar, I. Etxeberria-Otadui, R. Teodorescu, and F. Blaabjerg, "A stationary reference frame grid synchronization system for three-phase grid-connected power converters under adverse grid conditions," IEEE Transactions on Power Electronics, vol. 27, no. 1, pp. 99-112, Jan. 2012. 